Research Article

\title{
Numerical Steepest Descent Method for Hankel Type of Hypersingular Oscillatory Integrals in Electromagnetic Scattering Problems
}

\author{
Qinghua Wu 1 and Mengjun Sun \\ Hunan University of Science and Engineering, Yongzhou, 425199 Hunan, China \\ Correspondence should be addressed to Qinghua Wu; jackwqh@163.com \\ Received 13 April 2021; Accepted 15 June 2021; Published 22 July 2021 \\ Academic Editor: Ruben Specogna
}

Copyright (C) 2021 Qinghua Wu and Mengjun Sun. This is an open access article distributed under the Creative Commons Attribution License, which permits unrestricted use, distribution, and reproduction in any medium, provided the original work is properly cited.

We present a fast and accurate numerical scheme for approximating hypersingular integrals with highly oscillatory Hankel kernels. The main idea is to first change the integration path by Cauchy's theorem, transform the original integral into an integral on $[a$, $+\infty$ ], and then use the generalized Gauss Laguerre integral formula to calculate the corresponding integral. This method has the advantages of high-efficiency, fast convergence speed. Numerical examples show the effect of this method.

\section{Introduction}

In time-harmonic electromagnetic scattering, the following integral equations arise frequently $[1,2]$ :

$$
u^{s}(\rho)=-\frac{i k}{4} \int_{C} q\left(\rho^{\prime}\right) H_{0}^{(1)}\left(k\left|\rho-\rho^{\prime}\right|\right) d l^{\prime}
$$

where $H_{0}^{(1)}(x)$ is the Hankel function of order 0 and $q(x)$ is the unknown function.

For scatterers with sharp edges or corners, the unknown $q(x)$ should be sought in the following form [3]:

$$
q(x)=w(x) \phi(x), w(x)=(1-x)^{\alpha}(1+x)^{\beta},
$$

where $\phi(x)$ is smooth on $(-1,1)$, which leads to the following integral:

$$
\int_{-1}^{1} w(x) H_{0}^{(1)}(k \varphi(x)) \phi(x) d x .
$$

In addition, the following integral appears frequently in the fields of physics and engineering $[4,5]$ :

$$
I(f, w ; c, m, k)=f_{a}^{b} \frac{w(x) e^{i k x}}{(x-c)^{m+1}} f(x) d x, c \in(a, b),
$$

which is equivalent to the following form:

$$
I(f, w ; c, m, k)=\frac{1}{m !} \frac{d^{m}}{d c^{m}} \int_{a}^{b} \frac{w(x) e^{i k x}}{(x-c)} f(x) d x,
$$

where $w(x)=(x-a)^{\alpha}(b-x)^{\beta}$ and $\alpha>-1, \beta>-1$ and $f(x)$ is a given function. In order to ensure the existence of integrals, we need to assume that the $m-1$-th order derivative of $f$ is continuous and $f^{(m)}$ is Hölder continuous on $[a, b]$.

In the case $w(x) \equiv 1$ and $k=0$, many calculation methods have been proposed for (4), such as the Gauss-type method, the (composite) Newton-Cotes method, and others [6-10]. A popular method is using the Taylor formula and eliminates the singularity at $c$ by the following formula $[7,8,11]$ : 


$$
I(f, w ; c, m, k)=\int_{a}^{b} \frac{f(x)-\sum_{j=0}^{m} \frac{f^{(j)}(c)}{j !}(x-c)^{j}}{(x-c)^{m+1}} d x+\sum_{j=0}^{m} \frac{f^{(j)}(c)}{j !} f_{a}^{b} \frac{d x}{(x-c)^{m+1-j}}
$$

Although these methods are simple and generally feasible, they also have some disadvantages (e.g., numerical cancellations and computation of the higher derivatives). By using Chebyshev interpolants of $f(x)$, Hasegawa and Torii presented an efficiently uniform approximation algorithms for following integrals $[12,13]$.

$$
I(f, w ; c, m)=\int_{a}^{b} \frac{f(x)-\sum_{j=0}^{m} \frac{f^{(j)}(c)}{j !}(x-c)^{j}}{(x-c)^{m+1}} d x+\sum_{j=0}^{m} \frac{f^{(j)}(c)}{j !} f_{a}^{b} \frac{d x}{(x-c)^{m+1-j}} .
$$

However, it requires $O\left(N^{2}\right)$ operations, where $N-1$ is the degree of Chebyshev interpolants. There is a traditional method of calculating these integrals.

In the case $w(x) \equiv 1, k \gg 1$, the integrand is highly oscillatory. The traditional calculation methods of these integrals have the disadvantages of low efficiency and poor accuracy and will encounter difficulties (4). Xiang et al. presented an efficiently uniform approximation scheme for this case [14]. The principle is to establish the following new approximate formula:

$$
I(f ; c, m, k) \approx \frac{1}{m !} \frac{d^{m}}{d c^{m}} f_{-1}^{1} e^{i k x} \frac{p_{N}(x)-p_{N}(c)}{x-c} d x+\frac{1}{m !} \frac{d^{m}}{d c^{m}}\left(f(c) f_{-1}^{1} \frac{e^{i k x}}{x-c} d x\right),
$$

where $\quad p_{N}(x)=\left(a_{0} / 2\right) T_{0}(x)+\sum_{j=1}^{N-1} a_{j} T_{j}(x)+\left(a_{N} / 2\right) T_{N}(x)$ and $T_{j}(x)$ is the Chebyshev polynomial of the first kind.

Moreover, by rewriting $\left(p_{N}(x)-p_{N}(c)\right) /(x-c)$ in terms of $T_{j}(x)$ as follows:

$$
\frac{p_{N}(x)-p_{N}(c)}{x-c}=\sum_{j=0}^{N-1} d_{j} T_{j}(x)
$$

Equation (8) is transferred to

$$
I(f ; c, m, k) \approx \frac{1}{m !} \sum_{j=0}^{N-1}{ }^{\prime} M_{j}(k) \frac{d^{m}}{d c^{m}}\left(d_{j}\right)+\frac{1}{m !} \frac{d^{m}}{d c^{m}}\left(f(c) f_{-1}^{1} \frac{e^{i k x}}{x-c} d x\right),
$$

where the prime denotes the summation whose first term is halved and $M_{j}(k)=\int_{-1}^{1} T_{j}(x) e^{i k x} d x$ can be calculated using a recursive formula.

However, when $w(x)=(x+1)^{\alpha}(1-x)^{\beta}$ and $\alpha>-1, \beta>$ -1 , the recursion formula for $M_{j}(k)$ and $d_{j}$ is complicated. In [15], using the numerical steepest descent method, the following Cauchy principal value integral is calculated:

$$
f_{a}^{b} \frac{(x-a)^{a}(b-x)^{\beta} \ln (x-a)}{x-c} f(x) e^{i k x} d x
$$

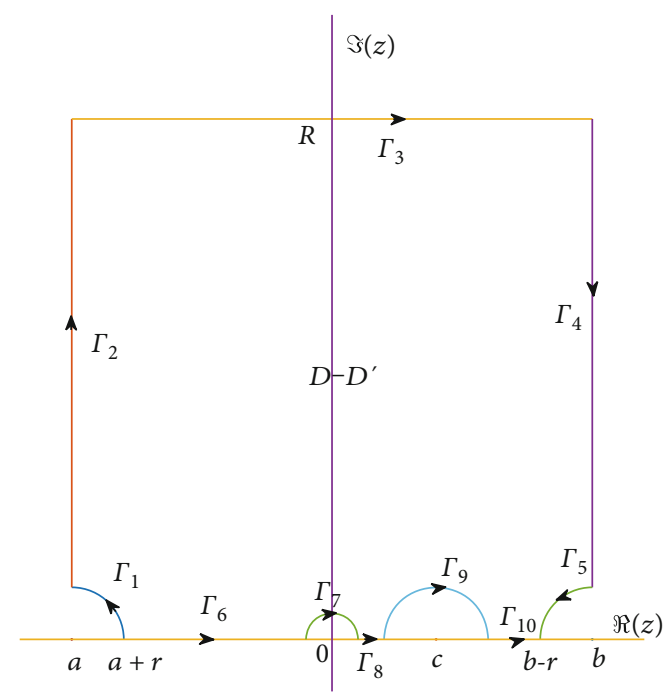

FIGURE 1: The integration paths.

Numerical results show that the calculation effect of the proposed method is better, but the situation of hypersingular is not considered in the article. In [16], using Hermite interpolation and a recurrence formula, Liu and Xiang present a method for calculating integrals (4) in combination with the numerical steepest descent method and gave the error analysis. For more details on singular integrals with oscillatory function, see Ref. [15, 17-22] and references therein.

In this paper, we study the direct steepest descent method for a class of hypersingular integral.

$$
I(f, w, G ; c, m, k)=f_{a}^{b} \frac{w(x) G(k x)}{(x-c)^{m+1}} f(x) d x, c \in(a, b),
$$

where $G(k x)=e^{i k x}$ or $H_{v}^{(1)}(k x), H_{v}^{(1)}$ denotes Hankel functions of the first kind of order $v(v \leq 1)$.

This paper is organized as follows: in Section 2, we review the basic formula for the steepest descent methods. In Section 3 , the performance of the method is demonstrated by numerical examples, which verify the efficiency and accuracy of the algorithm.

\section{Direct Steepest Descent Methods for Approximating the Integral (12)}

In this section, we focus on the steepest descent method on the evaluation of the following integral:

$$
I(f, w, H ; c, m, k)=f_{a}^{b} \frac{w(x) H_{v}^{(1)}(k x)}{(x-c)^{m+1}} f(x) d x, c \in(a, b) .
$$

In the case $G(k x)=e^{i k x}$, the method is similar except for deleting an integral path around 0 .

Set $D=\{z \in \mathbb{C} \mid a \leq \mathfrak{R}(z) \leq b, 0 \leq \mathfrak{I}(z) \leq R\}, D_{1}=\{z \in \mathbb{C}$ ||$z-a \mid \leq r, 0 \leq \arg (z-a) \leq \pi / 2\}, D_{2}=\{z \in \mathbb{C}|| z-b \mid \leq r, \pi$ $12 \leq \arg (z-a) \leq \pi\}, \quad D_{3}=\{z \in \mathbb{C}|| z-c \mid \leq r, 0 \leq \arg (z-c)$ $\leq \pi\}, D_{4}=\{z \in \mathbb{C}|| z \mid \leq r, 0 \leq \arg (z) \leq \pi\}$, and $D^{\prime}=D_{1} \bigcup$ 
$D_{2} \bigcup D 3 \bigcup D 4$, where $R$ is a large number and $r$ is small enough such that $D^{\prime} \subset D$ (see Figure 1).

Theorem 1. Suppose that $f(z)$ is an analytic function in the half-strip of the complex plane, $a \leq \mathfrak{R}(z) \leq b$, and $\mathfrak{\Im}(z) \geq 0$. If there are two constants $M$ and $k_{0}$ such that for $0 \leq k_{0}<k$

$$
\int_{a}^{b}|f(x+i R)| d x \leq M e^{k_{0} R}
$$

then the hypersingular integral with highly oscillatory kernels $I(f, w, H ; c, m, k)$ can be calculated by the following formula:

$$
I(f, w, H ; c, m, k)=\left\{\begin{array}{l}
S_{a}[f, w ; c, m, k]+S_{b}[f, w ; c, m, k]-\frac{1}{m !} \frac{d^{m}}{d c^{m}}\left(-i \pi w(c) f(c) H_{v}^{(1)}(k c)\right), \quad 0 \leq v<1, \\
S_{a}[f, w ; c, m, k]+S_{b}[f, w ; c, m, k]-\frac{1}{m !} \frac{d^{m}}{d c^{m}}\left(-i \pi w(c) f(c) H_{v}^{(1)}(k c)\right), \quad v=1, \\
+\frac{i}{k} \pi w(0) f(0) \lim _{r \rightarrow 0} H_{v}^{(1)}\left(k r e^{r \theta}\right)\left(k r e^{r \theta}\right)^{v},
\end{array}\right.
$$

where

$$
\begin{aligned}
S_{a}[f, w ; c, m, k]= & i^{\alpha+1} \int_{0}^{\infty} \frac{x^{\alpha}(b-a-i x)^{\beta}}{(a+i x-s)^{m+1}} H_{v}^{(1)} \\
& \cdot(k a+i k x) f(a+i x) d x, \\
S_{b}[f, w ; c, m, k]= & (-i)^{\beta+1} \int_{0}^{\infty} \frac{x^{\beta}(b-a+i x)^{\alpha}}{(b+i x-s)^{m+1}} H_{v}^{(1)} \\
& \cdot(k b+i k x) f(b+i x) d x .
\end{aligned}
$$

Proof. Since $\left(w(x) H_{v}^{(1)}(k x)\right) /\left((x-c)^{m+1}\right) f(x)$ is analytic in the region $D-D^{\prime}$, using the Cauchy's theorem, the following formula can be obtained:

$$
\int_{\Gamma_{1}+\Gamma_{2}+\Gamma_{3}+\Gamma_{4}+\Gamma_{5}-\Gamma_{6}-\Gamma_{7}-\Gamma_{8}-\Gamma_{9}-\Gamma_{10}} \frac{w(z) H_{v}^{(1)}(k x)}{(z-c)^{m+1}} f(z) d z=0 .
$$

Setting

$$
I_{j}=\int_{\Gamma_{j}} \frac{w(z) H_{v}^{(1)}(k x)}{(z-c)^{m+1}} f(z) d z, \quad j=1,2, \cdots, 10
$$

it derives that

$$
I_{1}+I_{2}+I_{3}+I_{4}+I_{5}-I_{7}-I_{9}=I_{6}+I_{8}+I_{10}
$$

Let $z-a=r e^{i \theta}, \theta \in[0, \pi / 2]$, then

$$
\begin{aligned}
\left|I_{1}\right|= & \mid i r \int_{0}^{\pi / 2} \frac{\left(r e^{i \theta}\right)^{\alpha}\left(b-a-r e^{i \theta}\right)^{\beta}}{\left(a+r e^{i \theta}-c\right)^{m+1}} H_{v}^{(1)} \\
& \cdot\left(k\left(a+r e^{i \theta}\right)\right) f\left(a+r e^{i \theta}\right) e^{i \theta} d \theta \mid \\
\leq & |r|^{1+\alpha} \int_{0}^{\pi / 2} \frac{\left|b-a-r e^{i \theta}\right|^{\beta}}{\left|a+r e^{i \theta}-c\right|^{m+1}} \mid H_{v}^{(1)}\left(k\left(a+r e^{i \theta}\right)\right) f \\
& \cdot\left(a+r e^{i \theta}\right) \mid d \theta \longrightarrow 0, a s r \longrightarrow 0 .
\end{aligned}
$$

Similarly, we have $\left|I_{5}\right| \longrightarrow 0$ as $r \longrightarrow 0$ and

$$
\left|I_{3}\right|=\left|\int_{a}^{b} \frac{(i R+x-a)^{\alpha}(b-i R-x)^{\beta}}{(i R+x-c)^{m+1}} H_{v}^{(1)}(k(i R+x)) f(i R+x) d x\right|
$$

For large arguments, the Hankel functions behave like an oscillatory complex exponential with a decaying amplitude:

$$
H_{v}^{(1)}(z) \sim \sqrt{\frac{2}{\pi z}} e^{i(z-(1 / 2) v \pi-(1 / 4) \pi)}, \quad-\pi<\arg z<\pi,|z| \longrightarrow \infty .
$$

As can be seen from the asymptotic behavior (22), for complex arguments with a positive imaginary part, the Hankel function decays exponentially, which follows that

$$
\left|I_{3}\right| \longrightarrow 0 \text {, as } R \longrightarrow+\infty \text {. }
$$


In addition, $I_{2}$ and $I_{4}$ can be represented as

$$
\begin{aligned}
I_{2}= & i^{\alpha+1} \int_{r}^{R} \frac{x^{\alpha}(b-a-i x)^{\beta}}{(a+i x-s)^{m+1}} H_{v}^{(1)}(k a+i k x) f(a+i x) d x, \\
I_{4}= & (-i)^{\beta+1} \int_{r}^{R} \frac{x^{\beta}(b-a+i x)^{\alpha}}{(b+i x-s)^{m+1}} H_{v}^{(1)}(k b+i k x) f(b+i x) d x, \\
I_{9}= & \frac{1}{m !} \frac{d^{m}}{d c^{m}}\left(\int_{\Gamma_{9}} \frac{(z-a)^{\alpha}(b-z)^{\beta}}{z-c} f(z) H_{v}^{(1)}(k z) d z\right) \\
= & \frac{1}{m !} \frac{d^{m}}{d c^{m}}\left(-i \int_{0}^{\pi}\left(c+1+r e^{i \theta}\right)^{\alpha}\left(b-c-r e^{i \theta}\right)^{\beta} f\right. \\
& \left.\cdot\left(c+r e^{i \theta}\right) H_{v}^{(1)}\left(k\left(c+r e^{i k \theta}\right)\right) d \theta\right) \\
\longrightarrow & \frac{1}{m !} \frac{d^{m}}{d c^{m}}\left(-i \pi w(c) f(c) H_{v}^{(1)}(k c)\right), a s r \longrightarrow 0 .
\end{aligned}
$$

Due to the Hankel function of order zero $H_{0}^{(1)}(z)$ having a logarithmic singularity at $z=0$ and Hankel functions of higher order having algebraic singularities of the form $1 / z^{v}$, $z \longrightarrow 0$ [23], we achieve

$$
\begin{aligned}
I_{7}= & \int_{\Gamma_{7}} \frac{(z-a)^{\alpha}(b-z)^{\beta}}{(k z)^{v}(z-c)^{m+1}} f(z) H_{v}^{(1)}(k z)(k z)^{v} d z \\
= & -i r \int_{0}^{\pi} \frac{\left(r e^{i \theta}-a\right)^{\alpha}\left(b-r e^{i \theta}\right)^{\beta}}{\left(k r e^{i \theta}\right)^{v}\left(r e^{i \theta}-s\right)^{m+1}} f\left(r e^{i \theta}\right) H_{v}^{(1)} \\
& \cdot\left(k\left(r e^{i k \theta}\right)\right)\left(k r e^{i \theta}\right)^{v} e^{i \theta} d \theta \\
& \longrightarrow\left\{\begin{array}{l}
0, \quad 0 \leq v<1, \\
-\frac{i}{k} \pi w(0) f(0) \lim _{r \longrightarrow 0} H_{v}^{(1)}\left(k r e^{r \theta}\right)\left(k r e^{r \theta}\right)^{v}, \quad v=1,
\end{array}\right.
\end{aligned}
$$

Thus, we obtain

$$
\begin{aligned}
I(f, w ; c, m, k)= & \lim _{r \longrightarrow 0, R \longrightarrow \infty} I_{1}+I_{2}+I_{3}+I_{4}+I_{5}-I_{7}-I_{9} \\
= & \begin{cases}S_{a}[f, w ; c, m, k]+S_{b}[f, w ; c, m, k]-\frac{1}{m !} \frac{d^{m}}{d c^{m}}\left(-i \pi w(c) f(c) H_{v}^{(1)}(k c)\right), & 0 \leq v<1, \\
S_{a}[f, w ; c, m, k]+S_{b}[f, w ; c, m, k]-\frac{1}{m !} \frac{d^{m}}{d c^{m}}\left(-i \pi w(c) f(c) H_{v}^{(1)}(k c)\right), & v=1, \\
+\frac{i}{k} \pi w(0) f(0) \lim _{r \longrightarrow 0} H_{v}^{(1)}\left(k r e^{r \theta}\right)\left(k r e^{r \theta}\right)^{v} .\end{cases}
\end{aligned}
$$

Formula (16) shows the integrals defined on $[0, \infty]$, and the integrand function decays exponentially. It can be calculated by the generalized Gauss-Laguerre quadrature rule. Suppose that $\left\{x_{l}^{(\alpha)}, w_{l}^{(\alpha)}\right\}_{l=1}^{N}$ and $\left\{x_{l}^{(\beta)}, w_{l}^{(\beta)}\right\}_{l=1}^{N}$ are the nodes and corresponding weights of generalized Gauss-Laguerre quadrature rule, where the generalized weight functions are defined as $x^{\alpha} e^{-x}$ and $x^{\beta} e^{-x}$, respectively. Then, integrals in (16) can be approximated by

$$
\begin{aligned}
S_{a}[f, w ; c, m, k] \approx & \left(\frac{i}{k}\right)^{\alpha+1} \sum_{l=1}^{N} w_{l}^{(\alpha)} \frac{\left(b-a-i x_{l}^{(\alpha)} / k\right)^{\beta}}{\left(a+i x_{l}^{(\alpha)} / k-c\right)^{m+1}} f \\
& \cdot\left(a+\frac{i x_{l}^{(\alpha)}}{k}\right) H_{v}^{(1)}\left(k a+i x_{l}^{(\alpha)}\right) e^{x_{l}^{(\alpha)}}, \\
S_{b}[f, w ; c, m, k] \approx & \left(-\frac{i}{k}\right)^{\beta+1} \sum_{l=1}^{N} w_{l}^{(\beta)} \frac{\left(b-a+i x_{l}^{(\beta)} / k\right)^{\alpha}}{\left(b+i x_{l}^{(\beta)} / k-c\right)^{m+1}} f \\
& \cdot\left(b+\frac{i x_{l}^{(\beta)}}{k}\right) H_{v}^{(1)}\left(k b+i x_{l}^{(\alpha)}\right) e^{x_{l}^{(\alpha)}}
\end{aligned}
$$

\section{Numerical Examples}

In this section, we illustrate the accuracy and efficiency of the method described in this paper with several numerical examples. All the calculations are done on Matlab R2016b, and all the exact values of the integrals are calculated by Maple with 32-digit arithmetic. The number of nodes used in generalized Gauss-Laguerre quadrature rule is set to 32. For the detail of the algorithm, see Appendix.

Example 1. Consider the calculation of the following Hadamard finite part integrals:

$$
I=f_{-1}^{1} \frac{e^{i k w}}{(x-s)^{2}} \cos (x) d x
$$

where "MP" indicates that the error has reached the accuracy of the machine in Matlab. Numerical results are illustrated in Table 1.

It can be seen from Table 1 that the calculated result has an error of order $O\left(10^{-15}\right)$, which indicates the accuracy of the proposed method is very high. 
TABLE 1: Relative errors of the direct steepest descent method for $I=f_{-1}^{1} \frac{e^{i k x}}{(x-c)^{2}} \cos (x) d x, c=0$.

\begin{tabular}{|c|c|c|c|c|}
\hline & \multicolumn{4}{|c|}{ Relative errors } \\
\hline & $k=10$ & $k=10^{2}$ & $k=10^{3}$ & $k=10^{5}$ \\
\hline & $7.1 \times 10^{-15}$ & MP & MP & MP \\
\hline Exact values & -31.44003099 & -314.16506211 & -3141.5917622 & -314159.2653 \\
\hline (Maple) & 497279 & 2116602 & 3182537 & 5859263 \\
\hline
\end{tabular}

TABLE 2: Relative errors of the direct steepest descent method for $I=f_{-1}^{1} \frac{\sin (k x)}{(x-c)^{3}} e^{x} d x, c=0$.

\begin{tabular}{|c|c|c|c|c|}
\hline & \multicolumn{4}{|c|}{ Relative errors } \\
\hline & $k=10$ & $k=10^{2}$ & $k=10^{3}$ & $k=10^{5}$ \\
\hline & $2.8 \times 10^{-14}$ & MP & MP & $1.2 \times 10^{-16}$ \\
\hline Exact values & -156.34590751 & 15707.459596 & -1570795.8045 & -15707963267.4 \\
\hline (Maple) & 00816 & 74907 & 23146 & 25343 \\
\hline
\end{tabular}

TABLE 3: Relative errors of the direct steepest descent method for $I=f_{-1}^{1} \frac{\sin (k x)}{x(x+1)^{0.5}(1-x)^{0.5}} d x .$.

\begin{tabular}{|c|c|c|c|c|}
\hline & \multicolumn{4}{|c|}{ Relative errors } \\
\hline & $k=10$ & $k=50$ & $k=100$ & $k=150$ \\
\hline & $\mathrm{MP}$ & $4.4 \times 10^{-16}$ & $8.9 \times 10^{-16}$ & $4.4 \times 10^{-16}$ \\
\hline Exact values & 3.352114873 & 2.8318697021 & 2.8986299106 & 2.9369583887 \\
\hline (Maple) & 80775 & 59874 & 88431 & 22875 \\
\hline
\end{tabular}

TABLE 4: Relative errors of the direct steepest descent method for $I=f_{-1}^{1} \frac{(x+1)^{-0.5}(1-x)^{-0.5}}{x-0.5} H_{1}^{(1)}(k x) d x$.

\begin{tabular}{lccrr}
\hline & & \multicolumn{2}{c}{ Relative errors } & $k=30$ \\
& $k=10$ & $k=20$ & $2.62 \times 10^{-16}$ & $5.88 \times 10^{-16}$ \\
Exact values & $1.02 \times 10^{-15}$ & $3.09 \times 10^{-16}$ & -0.2146909049936769 & 0.5217520845428135 \\
(Maple) & -0.8208250958210744 & -1.134059952837425 & $0.8319561170161881 \mathrm{i}$ & $0.1919435601126078 \mathrm{i}$ \\
\hline
\end{tabular}

Example 2. Consider the computation of the hypersingular integral

$$
I=f_{-1}^{1} \frac{e^{i k x}}{(x-c)^{2}} \cos (x) d x, c=0 .
$$

Its exact value can be expressed as follows [14]:

$$
\begin{aligned}
I= & \frac{i}{4} \frac{d^{m}}{d c^{m}}\left\{e ^ { s ( 1 + i k ) } \left[E_{1}((1-c)(1+i k))-E_{1}(-(1+s)(1+i k))\right.\right. \\
& +\ln (-1-i k)-\ln (1+i k)]+e^{-c(-1+i k)}\left[E_{1}((1+c)(-1+i k))\right. \\
& \left.\left.-E_{1}((1-c)(1-i k))+\ln (1-i k)-\ln (-1+i k)\right]\right\} .
\end{aligned}
$$

It can be seen from Table 2 that the calculation effect of the new method is very good, and as the frequency $k$ increases, the calculation accuracy also increases.

Example 3. Consider the computation of the Cauchy principle integral

$$
I=f_{-1}^{1} \frac{\sin (k x)}{(x-c)^{m+1}} e^{x} d x
$$

Its exact value can be expressed by the following formula:

$$
I=\pi k_{1} F_{2}\left(\frac{1}{2} ; 1, \frac{3}{2} ;-\frac{k^{2}}{4}\right) \text {, }
$$

where ${ }_{1} F_{2}(a ; b, c ; z)$ is the hypergeometric function. 
TABLE 5: Relative errors of the direct steepest descent method for $I=f_{-1}^{1} \frac{(x+1)^{-0.45}(1-x)^{-0.55}}{x-0.5} H_{1}^{(1)}(k x) d x$.

\begin{tabular}{|c|c|c|c|c|}
\hline & \multicolumn{4}{|c|}{ Relative errors } \\
\hline & $k=10$ & $k=20$ & $k=30$ & $k=40$ \\
\hline & $9.99 \times 10^{-16}$ & $3.21 \times 10^{-16}$ & $1.57 \times 10^{-16}$ & $5.8 \times 10^{-16}$ \\
\hline Exact values & -0.8021025230103994 & $-1.1913562 \mathrm{~s} 12289248$ & -0.2315525170424459 & 0.5759125632671623 \\
\hline (Maple) & $-1.0945266295060129 i$ & $0.02163694829164146 \mathrm{i}$ & $0.8911805394195018 \mathrm{i}$ & $0.1976701245353936 \mathrm{i}$ \\
\hline
\end{tabular}

function [ I ] = H1 ( a,b,c,k,N,alpha,beta )

$/ *$ int_a^ $\mathrm{a}^{\wedge} \mathrm{b}\left((\mathrm{x}-\mathrm{a})^{\wedge}\right.$ alpha $*(\mathrm{~b}-\mathrm{x})^{\wedge}$ beta $) /(\mathrm{x}-\mathrm{c}) \operatorname{Hanel}(\mathrm{kx}) \mathrm{dx} \mathrm{c}(\mathrm{a}, \mathrm{b})$.

$/ * \mathrm{~N}$ is the number of points used in generalized Gauss-Laguerre. [xa,wa] = GaussLaguerre( $\mathrm{N}$, alpha);

$/ *$ the generalized Gauss CLaguerre quadrature rule, $\mathrm{x}^{\wedge}$ alpha $\mathrm{e}^{\wedge}-\mathrm{x}[\mathrm{xb}, \mathrm{wb}]=$ GaussLaguerre $(\mathrm{N}$, beta);

/ $*$ the generalized Gauss CLaguerre quadrature rule, $x^{\wedge}$ beta $e^{\wedge}-\mathrm{x} j=1$;

$\mathrm{Sa}=\mathrm{wa} *\left((1 \mathrm{i} / \mathrm{k})^{\wedge}(\mathrm{alpha}+1) *(\mathrm{~b}-\mathrm{a}-1 \mathrm{i} * \mathrm{xa} / \mathrm{k}) . \wedge\right.$ beta. $/(\mathrm{a}-\mathrm{c}+1 \mathrm{i} * \mathrm{xa} / \mathrm{k}) . \wedge \mathrm{j} \ldots$

* $\operatorname{besselh}(1, \mathrm{k} * \mathrm{a}+1 \mathrm{i} * \mathrm{xa}) . * \exp (\mathrm{xa}))$;

$\mathrm{Sb}=\mathrm{wb} *\left((-1 \mathrm{i} / \mathrm{k})^{\wedge}(\mathrm{beta}+1) *(\mathrm{~b}-\mathrm{a}+1 \mathrm{i} * \mathrm{xb} / \mathrm{k}) . \wedge\right.$ alpha. $/(\mathrm{b}-\mathrm{c}+1 \mathrm{i} * \mathrm{xb} / \mathrm{k}) . \wedge \mathrm{j} \ldots$ *besselh $(1, \mathrm{k} * \mathrm{~b}+1 \mathrm{i} * \mathrm{xb}) . \exp (\mathrm{xb}))$;

$\mathrm{S} 7=-1 \mathrm{i} * \mathrm{pi} / \mathrm{k} / \mathrm{c} *(0-\mathrm{a})^{\wedge}$ alpha $*(\mathrm{~b}-0)^{\wedge}$ beta $* 2 * 1 \mathrm{i} / \mathrm{pi}$;

$\mathrm{S} 9=-1 \mathrm{i} * \mathrm{pi} *(\mathrm{c}-\mathrm{a})^{\wedge}$ alpha $*(\mathrm{~b}-\mathrm{c})^{\wedge}$ beta $*$ besselh $(1, \mathrm{k} * \mathrm{c})$;

$\mathrm{I}=\mathrm{Sa}+\mathrm{Sb}-\mathrm{S} 7-\mathrm{S} 9$

end

Code 1

Example 4. Consider the computation of the Cauchy principle integral

$$
I=f_{-1}^{1} \frac{\sin (k x)}{(x-c)^{3}} e^{x} d x, c=0
$$

For more general cases, Tables 3-5 show that the accuracy of the algorithm is very high, and it is very effective.

\section{Appendix}

The following is the main matlab code:

\section{Data Availability}

There is no data for this study.

\section{Conflicts of Interest}

The authors declare that there is no conflict of interests regarding the publication of this paper.

\section{Acknowledgments}

The design of the study and writing the manuscript is supported by the Natural Science Foundation of China under grant 11701170 and the Foundation for University Young Core Instructor from the Education Bureau of Hunan Province, China.

\section{References}

[1] J. L. Tsalamengas, "Quadrature rules for weakly singular, strongly singular, and hypersingular integrals in boundary integral equation methods," Journal of Computational Physics, vol. 303, pp. 498-513, 2015.

[2] L. Tsalamengas John, "Gauss-Jacobi quadratures for weakly, strongly, hyper- and nearly-singular integrals in boundary integral equation methods for domains with sharp edges and corners," Journal of Computational Physics, vol. 325, pp. 338-357, 2016.

[3] R. J. Mittra and S. W. Lee, Analytical Technique in the Theory of Guided Waves, Macmillan, 1971.

[4] G. Criscuolo, "A new algorithm for Cauchy principal value and Hadamard finite-part integrals," Journal of Computational and Applied Mathematics, vol. 78, no. 2, pp. 255-275, 1997.

[5] G. Monegato, "Definitions, properties and applications of finite-part integrals," Journal of Computational and Applied Mathematics, vol. 229, no. 2, pp. 425-439, 2009.

[6] G. Monegato, "Numerical evaluation of hypersingular integrals," Journal of Computational and Applied Mathematics, vol. 50, no. 1-3, pp. 9-31, 1994.

[7] D. Elliott and E. Venturino, "Sigmoidal transformations and the Euler-Maclaurin expansion for evaluating certain Hadamard finite-part integrals," Numerische Mathematik, vol. 77, no. 4, pp. 453-465, 1997.

[8] D. Kai, "A method for the practical evaluation of the Hilbert transform on the real line," Journal of Computational and Applied Mathematics, vol. 112, no. 1C2, pp. 45-53, 1999.

[9] J. Wu and W. Sun, "The superconvergence of Newton-Cotes rules for the Hadamard finite-part integral on an interval," Numerische Mathematik, vol. 109, no. 1, pp. 143-165, 2008. 
[10] T. Hasegawa and T. Torii, "An automatic quadrature for Cauchy principal value integrals," Mathematics of computation, vol. 56, no. 194, pp. 741-754, 1991.

[11] G. V. Milovanovi and M. M. Spalevi, "Quadrature rules with multiple nodes for evaluating integrals with strong singularities," Journal of Computational and Applied Mathematics, vol. 189, no. 1-2, pp. 689-702, 2006.

[12] T. Hasegawa and T. Torii, "Hilbert and Hadamard transforms by generalized Chebyshev expansion," Journal of Computational and Applied Mathematics, vol. 51, no. 1, pp. 71-83, 1994.

[13] T. Hasegawa, "Uniform approximations to finite Hilbert transform and its derivative," Journal of Computational and Applied Mathematics, vol. 163, no. 1, pp. 127-138, 2004.

[14] S. Xiang, C. Fang, and Z. Xu, "On uniform approximations to hypersingular finite-part integrals," Journal of Mathematical Analysis and Applications, vol. 435, no. 2, pp. 1210-1228, 2016.

[15] C. Fang, "Efficient methods for highly oscillatory integrals with weak and Cauchy singularities," International Journal of Computer Mathematics, vol. 93, no. 9, 2015.

[16] G. Liu and S. Xiang, "Clenshaw-Curtis-type quadrature rule for hypersingular integrals with highly oscillatory kernels," Applied Mathematics and Computation, vol. 340, pp. 251267, 2019.

[17] H. Wang and S. Xiang, "On the evaluation of Cauchy principal value integrals of oscillatory functions," Journal of Computational and Applied Mathematics, vol. 234, no. 1, pp. 95-100, 2010.

[18] H. Guo and S. Xiang, "An improved algorithm for the evaluation of Cauchy principal value integrals of oscillatory functions and its application," Journal of Computational and Applied Mathematics, vol. 280, pp. 1-13, 2015.

[19] T. Hasegawa and H. Sugiura, "Uniform approximation to finite Hilbert transform of oscillatory functions and its algorithm," Journal of Computational and Applied Mathematics, vol. 358, pp. 327-342, 2019.

[20] G. Rządkowski and E. Tohidi, "A fourth order product integration rule by using the generalized Euler-Maclaurin summation formula," Journal of computational and applied mathematics, vol. 335, pp. 334-348, 2018.

[21] H. Wang and H. Kang, "Numerical methods for two classes of singularly oscillatory Bessel transforms and their error analysis," Journal of Computational and Applied Mathematics, vol. 371, p. 112604, 2020.

[22] Z. Xu, H. Geng, and C. Fang, "Asymptotics and numerical approximation of highly oscillatory Hilbert transforms," Applied Mathematics and Computation, vol. 386, p. 125525, 2020.

[23] M. Abramowitz and I. A. Stegun, Handbook of Mathematical Functions, National Bureau of Standards, Washington, DC, 1964. 